\title{
An Alternative Paper Based Tissue Washing Method for Mass Spectrometry Imaging: Localized Washing and Fragile Tissue Analysis
}

\author{
Erika R. Amstalden van Hove, ${ }^{1}$ Donald F. Smith, ${ }^{1}$ Lara Fornai, ${ }^{1}$ Kristine Glunde, ${ }^{3}$ \\ Ron M. A. Heeren ${ }^{1,2}$ \\ ${ }^{1}$ FOM Institute AMOLF, Science Park 104, 1098 XG Amsterdam, The Netherlands \\ ${ }^{2}$ The Netherlands Proteomics Centre, Utrecht University, H.R. Kruytgebouw, Utrecht, The Netherlands \\ ${ }^{3}$ JHU In Vivo Cellular and Molecular Imaging Center, The Russell H. Morgan Department of Radiology and Radiological \\ Sciences, Johns Hopkins University School of Medicine, Baltimore, MD, USA
}

\begin{abstract}
Surface treatment of biological tissue sections improves detection of peptides and proteins for mass spectrometry imaging. However, liquid surface treatments can result in diffusion of surface analytes and fragile tissue sections can be easily damaged by typical washing solvents. Here, we present a new surface washing procedure for mass spectrometry imaging. This procedure uses solvent wetted fiber-free paper to enable local washing of tissue sections for mass spectrometry imaging and tissue profiling experiments. In addition, the method allows fragile tissues that cannot be treated by conventional washing techniques to be analyzed by mass spectrometry imaging.
\end{abstract}

Key words: Imaging Mass Spectrometry, Washing, Peptides and Proteins, MALDI, SIMS

\section{Introduction}

$\mathrm{T}$ he quality of mass spectrometry (MS) imaging results depends heavily on the choice and execution of sample preparation methods. Specific molecular classes often require different sample preparation treatments, such as optimized washing steps and the correct choice of matrix for matrix assisted laser desorption ionization (MALDI). Several washing methods have been tested, optimized, and have become standard elements in many MS imaging workflows.[1-5] Popular washing methods include immersion of the sample in washing solution or pipette application of the solution onto the sample and subsequent removal by pipette. However, such washing steps often lead to undesired diffusion of molecules over the tissue surface. Delicate biological sections can also be severely damaged when washed with harsh solvents.

Blotting techniques have been applied to transfer intact proteins from biological sections onto polyvinylidene difluoride (PVDF) membranes, which are then measured in an indirect imaging experiment [6-8]. The "molecular

Correspondence to: Ron M. A. Heeren; e-mail: heeren@amolf.nl scanner" has been used to extract and digest proteins from 1D and 2D SDS-PAGE gels and from tissues onto capture membranes, while keeping their spatial organization [9-11].

Here, we present a surface cleaning method which also relies on the contact between biological material and a wetted fiberfree paper. Unlike the above-mentioned blotting techniques, here the biological tissue is directly analyzed by a MS imaging experiment. The solutions used in the surface cleaning are the same solutions already established for MS imaging sample preparation $[1-5,12]$. The use of paper for surface cleaning allows its application on selected sample areas, allowing the simultaneous comparison of washed and unwashed areas on a single tissue sample. This gentle method allows surface cleaning of fragile biological samples, which cannot be washed by pipette or immersion methods.

\section{Experimental}

Rat heart and rat brain from male rats (type WU) and mouse brain from a female mouse (type 9CFW-1) were purchased from Harlan Laboratories (Boxmeer, The 
Netherlands). Rat heart and brain were sectioned at $10 \mu \mathrm{m}$ thickness on a cryo-microtome (Microm International, Walldorf, Germany) and thaw mounted on indiumtin-oxide coated glass slides (ITO, 4-8 $\Omega$ resistance; Delta Technologies, Stillwater, MN, USA). Adjacent $12 \mu \mathrm{m}$ thick mouse brain sections were collected for comparison of washing methods and thaw mounted on ITO slides. All samples were stored at $-80{ }^{\circ} \mathrm{C}$ prior to use and dried in a vacuum desiccator for 30 min prior to washing, matrix coating, and MS analysis.

\section{Fiber Free Paper Washing Procedure}

The fiber-free paper washing procedure uses a common laboratory wipe (KIMTECH SCIENCE* Precision Wipes; Kimberly-Clark, Roswell, GA, USA), which is wetted with the wash solvent of choice and carefully placed on top of the tissue section. The paper is cut to a size slightly larger than the tissue section to allow for full coverage, or can be cut to any size for localized washing. Typically, a solvent volume of 100 $200 \mu \mathrm{L}$ is sufficient to completely wet the paper with a pipette. Alternatively, the papers can be soaked in the wash solvent before application, though care should be taken that the paper is not too saturated, as this can cause flooding of the tissue section. Contact time is typically $30-60 \mathrm{~s}$, and depends on the washing type and solvent of choice. Unlike submersion and pipette washing methods, volatile washing solvents (such as alcohols) will evaporate from the paper, which does not allow extensive contact periods ( $>60 \mathrm{~s})$. Care should be taken when removing the wetted paper from the tissue section in order to reduce smearing effects from the application and lifting process.

Rat heart was washed twice with fiber-free paper wetted with $70 \%$ cold ethanol, each for 1 minute. The paper used to wash the rat heart was saved and imaged by metal enhanced secondary-ion mass spectrometry (MetA-SIMS). A rat brain section was locally washed with paper strips wetted with $70 \%$ cold ethanol for 1 minute per strip. A mouse brain section was washed with isopropanol $(70 \%$ and $95 \%$, in $\mathrm{H}_{2} \mathrm{O} ; 30 \mathrm{~s}$ each) for comparison with conventional washing procedures for the enhancement of protein signals.

\section{Immersion Washing Procedure}

The immersion washing procedure was adapted from Seeley et al. [4]. The ITO slide with the tissue section was immersed in a bath of the wash solvent of choice in a Petri

\section{(a)}

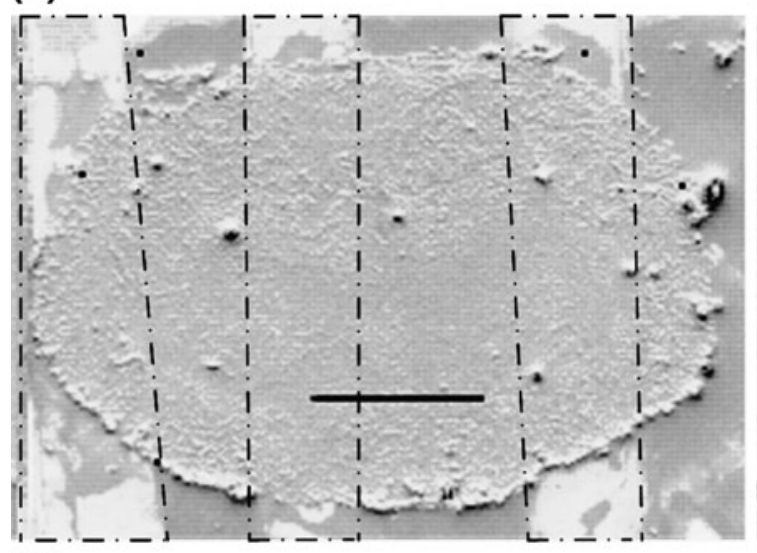

(b)

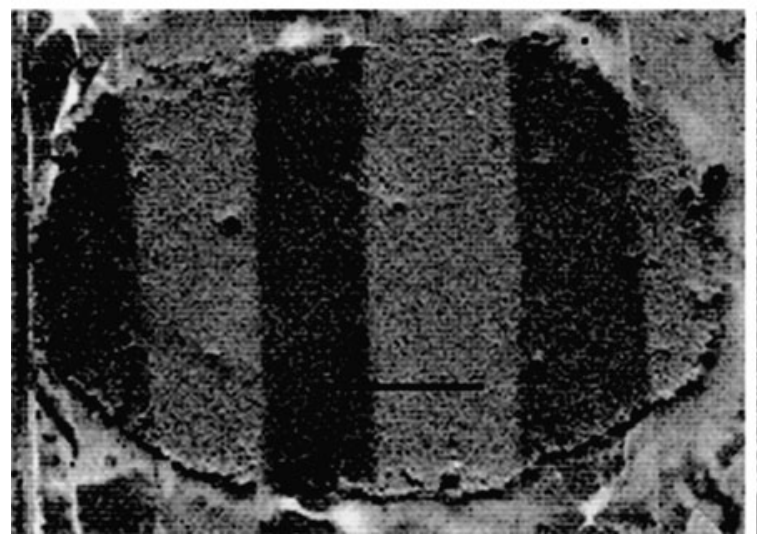

(c)

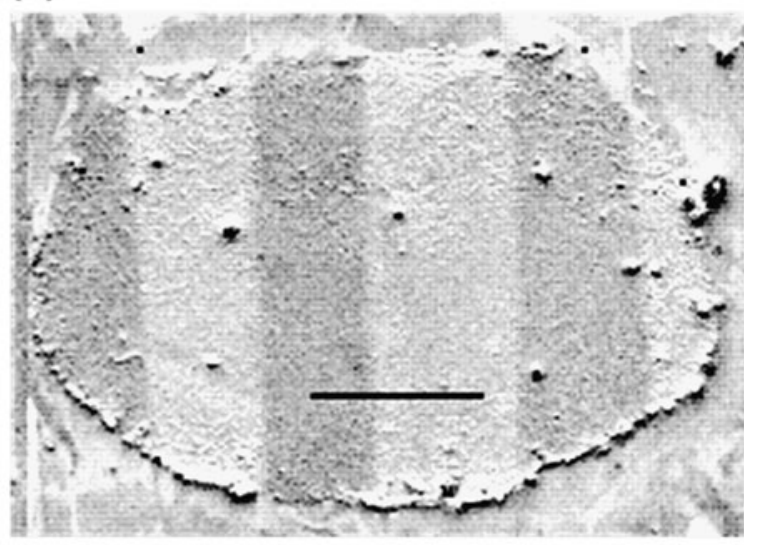

(d)

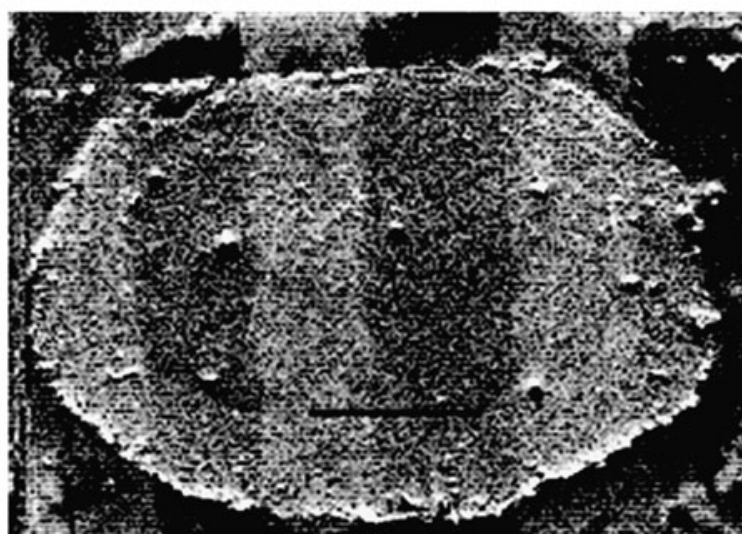

Figure 1. MetA-SIMS MS imaging experiment of a selectively washed rat brain section. The areas enclosed by the dotted lines in (a) indicate the areas selectively washed by wetted strips of paper. (a) Total ion image, (b) $\mathrm{Na}^{+}(\mathrm{m} / z 22.9)$, (c) $\mathrm{K}^{+}(\mathrm{m} / z \mathbf{z} 8.9)$, and (d) phosphocholine $(\mathrm{m} / \mathrm{z}$ 184). Scale bar $=1 \mathrm{~cm}$ 
dish (typically $50 \mathrm{~mL}$ solvent). No swirling or mixing was performed. A mouse brain section was immersed in isopropanol ( $70 \%$ and $95 \%$, in $\mathrm{H}_{2} \mathrm{O} ; 30 \mathrm{~s}$ each) for comparison with wetted paper and pipette washing. A mouse brain section spotted with permanent markers was immersed in $70 \%$ ethanol (in $\mathrm{H}_{2} \mathrm{O}$ ) for $60 \mathrm{~s}$ to assess the degree of diffusion of small molecules.

\section{Pipette Washing Procedure}

The pipette washing procedure was adapted from Lemaire et al. [2]. A volume of $\sim 200 \mu \mathrm{L} / \mathrm{cm}^{2}$ was loaded into a pipette and rinsed over the tissue section while holding the ITO slide at an angle to allow the solvent to efficiently drain. A mouse brain section was rinsed with isopropanol $(70 \%$ and (a)

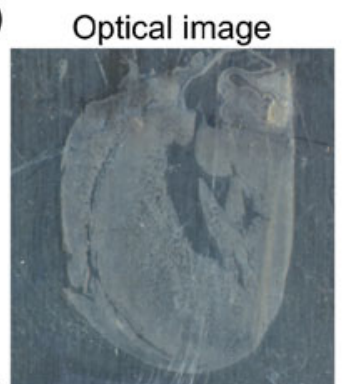

(b)

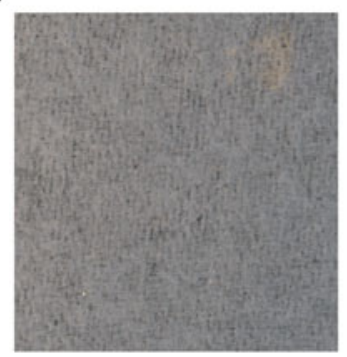

(c)

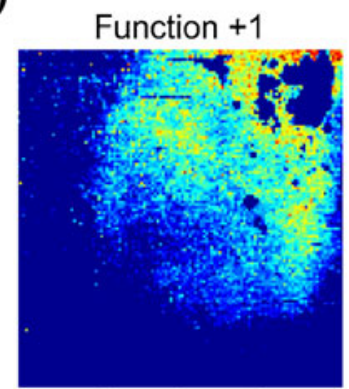

Function -1

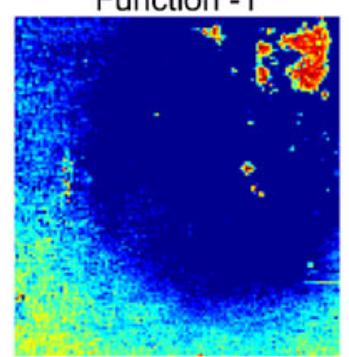

$\mathrm{Na}^{+}$
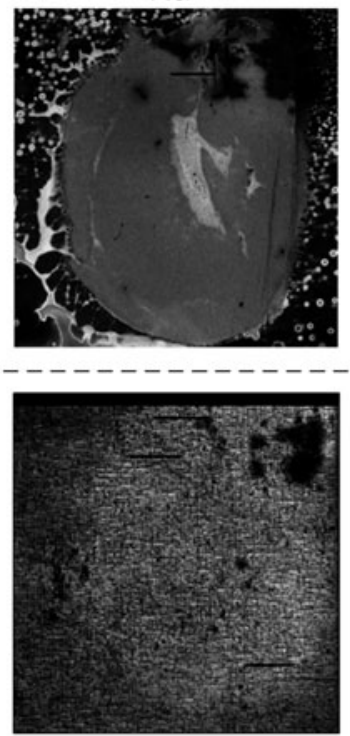

(d)
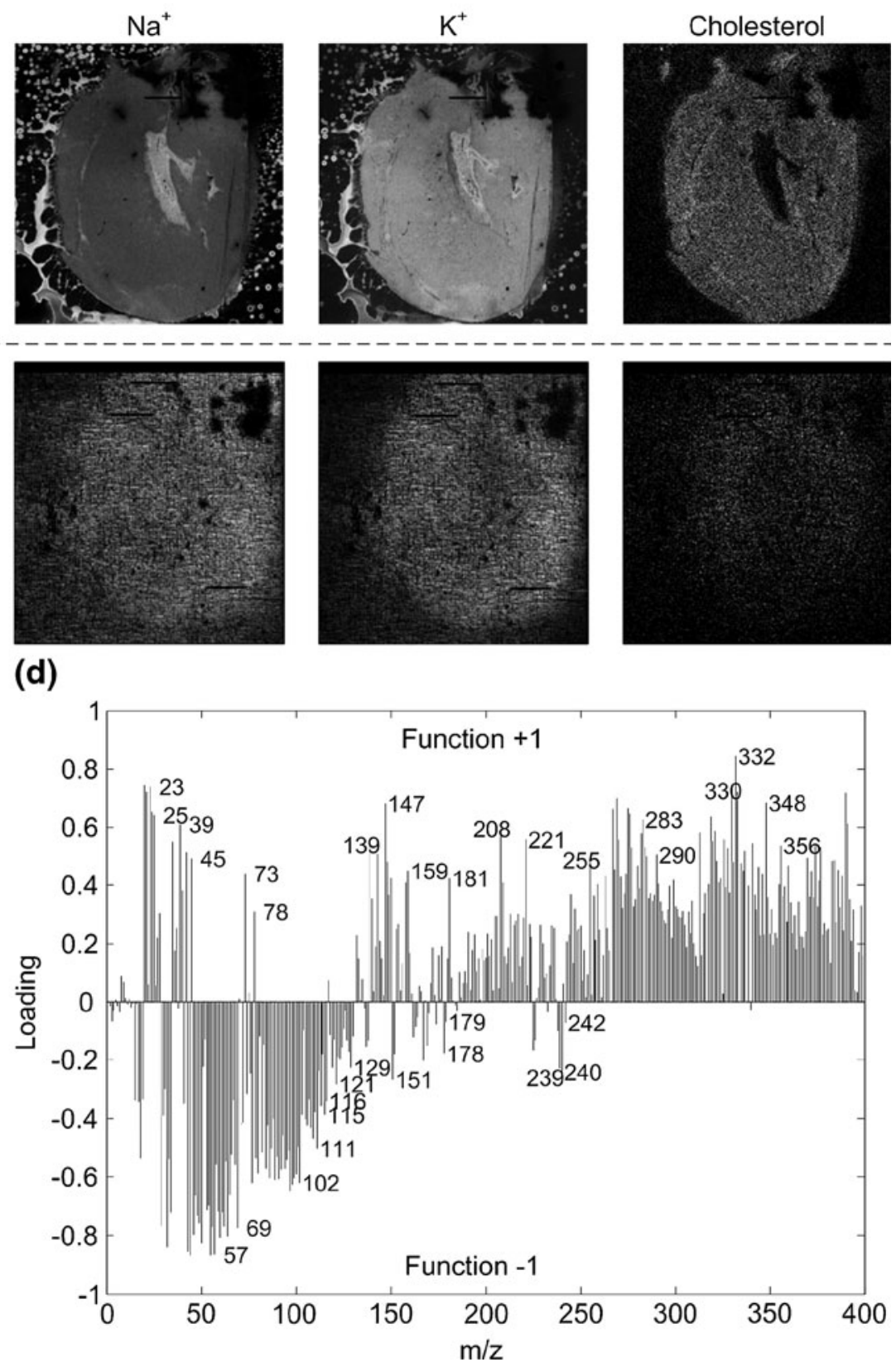

Figure 2. MetA-SIMS of a rat heart and the cleaning paper from a $70 \%$ ethanol treatment. (a) lon selected images from the rat heart and (b) corresponding images of the same species from the washing paper. (c) Image of the first principal component of the MetA-SIMS spectra acquired from the paper used to wash the heart tissue and (d) the corresponding loading spectrum. Dominant species from the area in contact with the rat heart section show removal of salt cations, silicone contaminants such as polydimethylsiloxane, $\mathrm{m} / z 73\left(\mathrm{C}_{3} \mathrm{H}_{9} \mathrm{Si}^{+}\right)$and $\mathrm{m} / z 147\left(\mathrm{C}_{5} \mathrm{H}_{15} \mathrm{OSi}_{3}{ }^{+}\right)$, and a mounting compound (Optimal Cutting Temperature compound) related peak at $\mathrm{m} / \mathrm{z} 332$ 
$95 \%$, in $\mathrm{H}_{2} \mathrm{O} ; 200 \mu \mathrm{L}$ each) for comparison with wetted paper and immersion washing.

\section{Metal Deposition}

For MetA-SIMS, a $1 \mathrm{~nm}$ gold layer was sputter-coated onto the locally washed rat brain section and the paper used to wash the heart section following the protocol described in reference [3].

\section{Matrix Deposition}

Sinapinic acid $(25 \mathrm{mg} / \mathrm{mL}$ in $50 \%$ acetonitrile $/ 0.1 \%$ trifluoroacetic acid) was used for protein MS imaging of the mouse brain sections washed with isopropanol. The matrix was applied using a vibrational spray coater (ImagePrep; Bruker Daltonics, Bremen, Germany). For the comparison of diffusion for small molecules on mouse brain tissues subjected to different washing procedures, $\alpha$-cyano-4hydroxycinnamic acid $(10 \mathrm{mg} / \mathrm{mL}$ in $50 \%$ acetonitrile $/ 0.1 \%$ trifluoroacetic acid) was used and applied using a vibrational spray coater, as described above.

\section{MS Imaging}

SIMS experiments were performed on a Physical Electronics (Eden Prairie, MN, USA) TRIFT II time-of-flight secondary ion mass spectrometer with an $\mathrm{Au}^{1+}$ primary ion beam. The data acquisition and visualization were performed by WinCadence ver. 4.4.0.17

MALDI experiments were performed on an Ultraflex III time-of-flight mass spectrometer (Bruker Daltonik GmbH, Bremen, Germany), with an accumulation of 1000 shots per position for proteins and 700 shots for small molecules with a raster size of $200 \mu \mathrm{m}$.

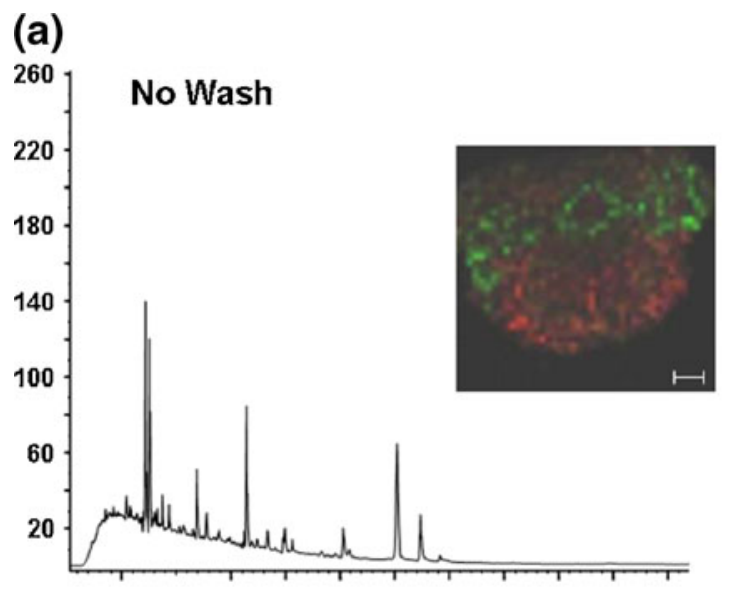

(b)
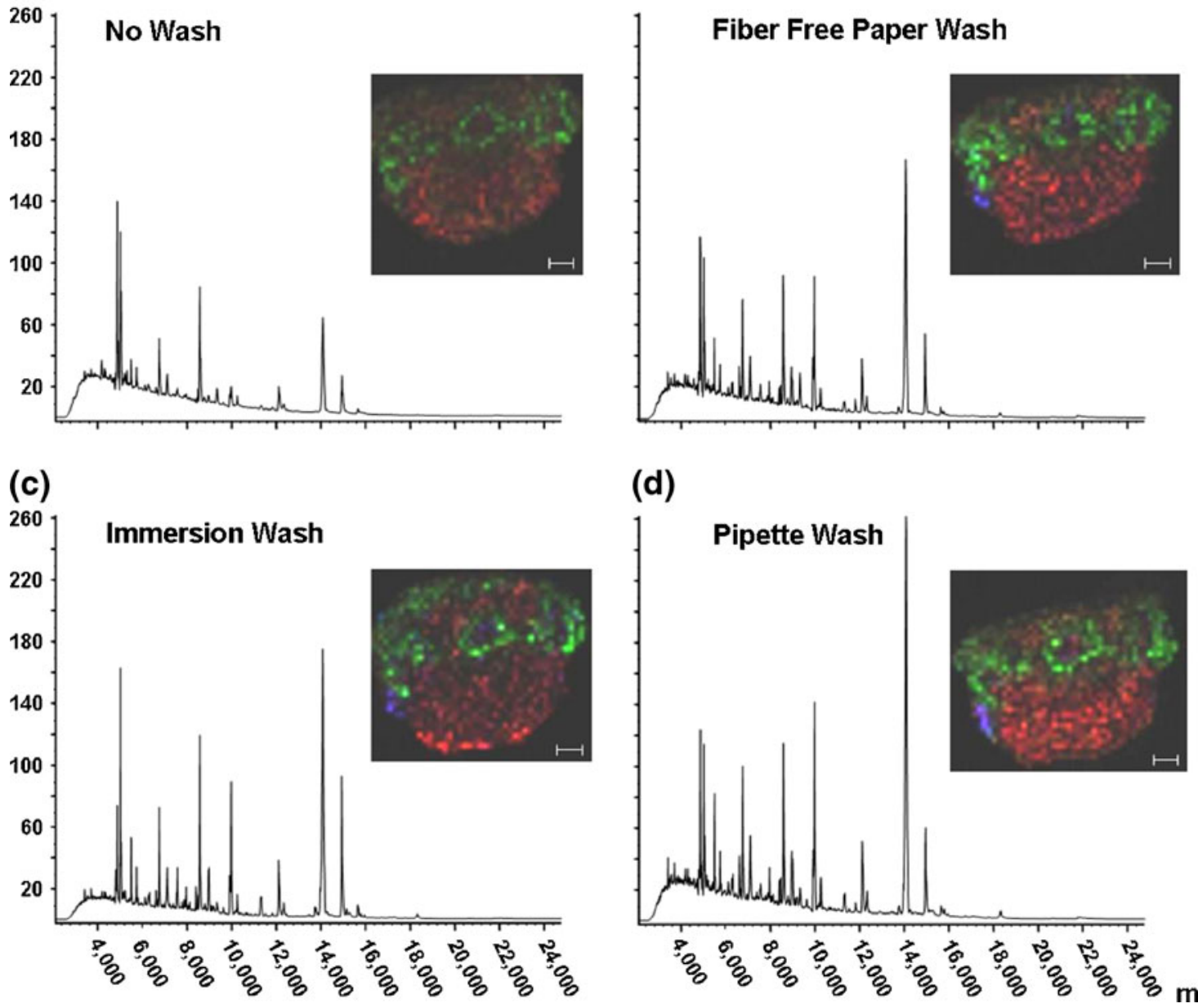

(d)

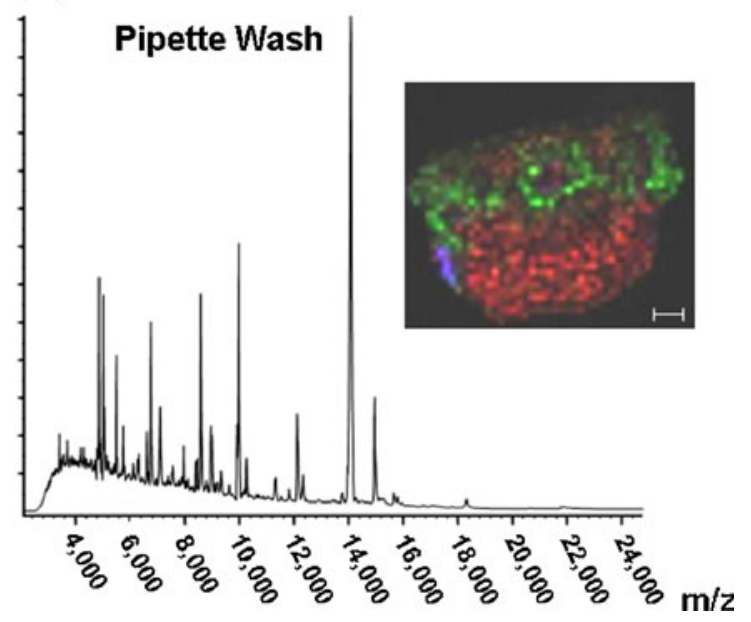

Figure 3. Spectral comparison of different washing methods of mouse brain sections using isopropanol $(70 \%$ and $95 \%$ in $\mathrm{H}_{2} \mathrm{O}$, $30 \mathrm{~s}$ each, or $200 \mu \mathrm{L}$ each by the pipette method)). The baseline subtracted, average mass spectra (over the entire image) are shown. (a) No washing step, (b) wetted fiber free paper wash, (c) immersion wash, (d) pipette wash. Inset images show three selected protein ions (blue: $\mathrm{m} / \mathrm{z} 13,751 \pm 10$, green: $\mathrm{m} / \mathrm{z} 6755 \pm 10$, and red: $\mathrm{m} / \mathrm{z} 7093 \pm 10$ ), which show that the spatial organization is retained by all washing methods. Scale bar $=1 \mathrm{~mm}$ 


\section{Results and Discussion}

Tissue washing has been shown to improve the spectral wealth and quality for intact proteins, as well as improving peptide detection [1-5]. Here, a new method is presented that uses a fiber free paper, wetted with the wash solvent of choice, which allows localized washing and presents a number unique advantages over conventional methods. Samples deposited on substrates that cannot be washed by conventional methods due to sample mounting (e.g., wholebody animal section deposited on double sided adhesive tape) can be washed with this method, as well as fragile sections that may be damaged by conventional washing procedures.

The use of wetted paper allows surface cleaning of selected areas of a tissue section. Thus, analytes detected with and without surface washing can be compared on one tissue section. Paper strips wetted with $70 \%$ ethanol were applied on different parts of a rat brain and the section was measured by MetA-SIMS. Figure 1 shows the total ion image (Figure 1a) and mass selected ion images of sodium $(\mathrm{m} / \mathrm{z} 22.9)$ (Figure 1b), potassium $(\mathrm{m} / \mathrm{z}$ 38.9) (Figure 1c), and phosphocholine $(\mathrm{m} / \mathrm{z}$ 184) (Figure 1d) from the localized washing experiment. The washed areas show a decrease in signal intensity for salt cations such as sodium and potassium, and an increase in signal intensity for small organic molecules such as phosphocholine $(\mathrm{m} / z$ 184).

The decrease in relative abundance of (salt) cations is attributed to the removal of these species from the tissue section into the wetted paper. Evidence of this migration is presented in Figure 2, where an entire rat heart section was cleaned once with wetted paper (cold 70\% ethanol, $60 \mathrm{~s}$ ). The analysis of the paper surface by MetA-SIMS shows that $\mathrm{Na}^{+}$and $\mathrm{K}^{+}$were blotted from the tissue section onto the paper (Figure 2b). The signal enhancement of certain ions after this localized surface cleaning procedure is attributed to reduced ion suppression resulting from the removal of the salts. The method does result in transfer of some organic molecules, such as cholesterol, to the washing paper. Principal component analysis (PCA) was performed on the MetA-SIMS data acquired from the paper surface. The first principal component spectra in Figure 2c demonstrates the removal of silicone contaminates at $\mathrm{m} / z 73$ and 147 [13], which can be detrimental to SIMS experiments.

In order to compare washing methods, serial mouse brain sections were freshly prepared and submitted to three washing protocols with isopropanol $(70 \%$ and $95 \%$ in $\mathrm{H}_{2} \mathrm{O}$ ), coated with sinapinic acid MALDI matrix and imaged. Figure 3 shows the average, baseline subtracted, mass spectra from the MS imaging runs and an overlay of three selected protein ion images. Without a washing procedure (Figure 3a), proteins are still detected from the tissue, but fewer peaks are observed. All three washing procedures improve the protein ion detection and retain their spatial distribution. We conclude that the fiber-free wetted paper method provides similar spectral results when com- pared with accepted standard washing procedures for proteins. The spatial distribution of intact proteins has been show to be affected little by washing procedures prior to MS imaging [4] (Figure 3).

\section{Conclusions}

The method described in this application note is a simple, effective method for the washing of biological tissue sections for MALDI and SIMS mass spectrometry imaging. The method uses a common laboratory paper wetted with washing solvent that is blotted onto the tissue sample surface. Fragile tissue sections, not amenable to conventional wet washing methods (thus resulting in poor MALDI MS imaging data quality), can be cleaned with this method and show good MALDI MS imaging spectral and image quality. Localized areas of tissue sections can be selectively washed, and SIMS imaging of a selectively washed tissue section shows increase in signal intensity of small organic molecules and a decrease in signal intensity of salt cations in the washed areas. Contaminant agents such as PDMS can be removed from the analyte surface, improving the data quality. The surface cleaning procedure described here is a reproducible, reliable, and efficient method that can be applied in any type of sample preparation for SIMS and MALDI-MS imaging.

\section{Acknowledgments}

This work is part of the research program of the Stichting voor FundamenteelOnderzoek der Materie (FOM), which is financially supported by the Nederlandse organisatie voor Wetenschappelijk Onderzoek (NWO). The authors acknowledge partial support for this work by the National Institutes of Health (NIH) grant R01 CA134695 and the Netherlands Proteomics Centre.

\section{Open Access}

This article is distributed under the terms of the Creative Commons Attribution Noncommercial License which permits any noncommercial use, distribution, and reproduction in any medium, provided the original author(s) and source are credited.

\section{References}

1. Schwartz, SA., Reyzer, M.L., Caprioli, R.M.: Direct Tissue Analysis Using Matrix-Assisted Laser Desorption/Ionization Mass Spectrometry: Practical Aspects of Sample Preparation. J. Mass Spectrom. 38(7), 699-708 (2003)

2. Lemaire, R., Wisztorski, M., Desmons, A., Tabet, J.C., Day, R., Salzet, M., Fournier, I.: MALDI-MS Direct Tissue Analysis of Proteins: Improving Signal Sensitivity Using Organic Treatments. Anal. Chem. 78(20), 7145-7153 (2006)

3. Altelaar, A.F.M., Luxembourg, S.L., McDonnell, L.A., Piersma, S.R., Heeren, R.M.A.: Imaging Mass Spectrometry at Cellular Length Scales. Nat. Protocols 2(5), 1185-1196 (2007)

4. Seeley, E.H., Oppenheimer, S.R., Mi, D., Chaurand, P., Caprioli, R.M.: Enhancement of Protein Sensitivity for MALDI Imaging Mass 
Spectrometry After Chemical Treatment of Tissue Sections. J. Am. Soc. Mass Spectrom. 19(8), 1069-1077 (2008)

5. Kaletaş, B.K., van der Wiel, I.M., Stauber, J., Lennard, J.D., Güzel, C., Kros, J.M., Luider, T.M., Heeren, R.M.: A. Sample Preparation Issues for Tissue Imaging by Imaging MS. Proteomics 9(10), 2622-2633 (2009)

6. Caprioli, R.M., Farmer, T.B., Gile, J.: Molecular Imaging of Biological Samples: Localization of Peptides and Proteins Using MALDI-TOF MS. Anal. Chem. 1997 69(23), 4751-4760 (2009)

7. Chaurand, P., Schwartz, S.A., Caprioli, R.M.: Imaging Mass Spectrometry: A New Tool to Investigate the Spatial Organization of Peptides and Proteins in Mammalian Tissue Sections. Curr. Opin. Chem. Biol. 6 (5), 676-681 (2002)

8. Chaurand, P., Stoeckli, M., Caprioli, R.M.: Direct Profiling of Proteins in Biological Tissue Sections by MALDI Mass Spectrometry. Anal. Chem. 71(23), 5263-5270 (1999)
9. Muller, M., Gras, R., Appel, R.D., Bienvenut, W.V., Hochstrasser, D. F.: Visualization and Analysis of Molecular Scanner Peptide Mass Spectra. J. Am. Soc. Mass Spectrom. 13(3), 221-231 (2002)

10. Rohner, T.C., Staab, D., Stoeckli, M.: MALDI Mass Spectrometric Imaging of Biological Tissue Sections. Mech. Ageing Dev. 126(1), 177-185 (2005)

11. Luxembourg, S.L., Vaezaddeh, A.R., Amstalden, E. R., Zimmermann-Ivol, C.G., Hochstrasser, D.F., Heeren, R.M.: A. The Molecular Scanner in Microscope Mode. Rapid Commun. Mass Spectrom. 20(22), 3435-3442 (2006)

12. Todd, P.J., Schaaff, T.G., Chaurand, P., Caprioli, R.M.: Organic Ion Imaging of Biological Tissue with Secondary Ion Mass Spectrometry and Matrix-Assisted Laser Desorption/Ionization. J. Mass Spectrom. 36 (4), 355-369 (2001)

13. Vickerman, J.C., and Briggs, D.: ToF-SIMS Surface Analysis by Mass Spectrometry. IM Publications and Surface Spectra Ltd: Chichester, Manchester, 2001. 